\title{
Determination of Elemental Composition of Shale Rocks by Laser Induced Breakdown Spectroscopy (LIBS)
}

\author{
Hervé K. Sanghapi ${ }^{1}$, Jinesh Jain ${ }^{2 *}$, Alexander Bol'shakov ${ }^{3}$, Christina Lopano ${ }^{2}$, Dustin McIntyre ${ }^{4}$, Richard Russo ${ }^{3}$ \\ ${ }^{1}$ Institute for Clean Energy Technology, Mississippi State University, Starkville, MS 39759, USA \\ ${ }^{2}$ U.S. Department of Energy, National Energy Technology Laboratory, Pittsburgh, PA 15236, USA \\ ${ }^{3}$ Applied Spectra, Inc. Fremont, CA 94538, USA \\ ${ }^{4}$ U.S. Department of Energy, National Energy Technology Laboratory, Morgantown, WV 26507, USA \\ *corresponding author
}

\begin{abstract}
In this study laser induced breakdown spectroscopy (LIBS) is used for elemental characterization of outcrop samples from the Marcellus Shale. Powdered samples were pressed to form pellets and used for LIBS analysis. Partial least squares regression (PLS-R) and univariate calibration curves were used for quantification of analytes. The matrix effect is substantially reduced using the partial least squares calibration method. Predicted results with LIBS are compared to ICP-OES results for $\mathrm{Si}, \mathrm{Al}, \mathrm{Ti}, \mathrm{Mg}$, and $\mathrm{Ca}$. As for $\mathrm{C}$, its results are compared to those obtained by a carbon analyzer. Relative errors of the LIBS measurements are in the range of 1.7 to $12.6 \%$. The limits of detection (LOD) obtained for $\mathrm{Si}, \mathrm{Al}, \mathrm{Ti}, \mathrm{Mg}$ and $\mathrm{Ca}$ are 60.9, 33.0, 15.6, 4.2 and $0.03 \mathrm{ppm}$, respectively. An LOD of 0.4wt\% was obtained for carbon. This study shows that the LIBS method can provide a rapid analysis of shale samples and can potentially benefit depleted gas shale carbon storage research.
\end{abstract}

\section{Introduction}

Shales are of interest in geochemical and geological investigations because they may host petroleum and natural gas. For example, oil shale contains solid bituminous material (called kerogen) that yields substantial amount of oil and combustible gas upon destructive distillation. The Marcellus shale deposits in eastern United States are well known for a large amount of natural gas distribution sorbed in the shale and in cracks and pores. Organic-rich shale formations that have been depleted of hydrocarbons through a period of primary production are potential candidates for geologic storage of $\mathrm{CO}_{2}$ [1][2], accompanied by enhanced gas recovery (EGR). Marcellus shale is an ideal material to study elemental profile because: 1) both $\mathrm{CO}_{2}$ and 
natural gas $\left(\mathrm{CH}_{4}\right)$ adsorption/desorption seem to have a correlation with mineral composition of the rocks [3], 2) the rocks that contain higher amounts of carbon contents (organic material) have greater ability to generate natural gas and potentially a greater capacity of $\mathrm{CO}_{2}$ storage [4], and 3) environmental issues associated with shale retorting requires substantial monitoring and control of waste products [5]. Thus there are a number of applications that would benefit from in situ and/or rapid knowledge of the elemental composition of the shale rock.

The analytical techniques[6] used for elemental analysis of shale are instrumental neutron activation analysis (INNA), X-ray fluorescence (XRF), and inductively coupled plasmaoptical emission spectroscopy (ICP-OES). INNA needs a large neutron reactor for irradiation and the method is inherently slow. Although XRF is a relatively fast technique, the analysis generally requires a larger sample size and the technique is known [7] to achieve poor detection limits, particularly for light elements. ICP-OES [8] is commonly used for determination of elemental composition of shale. This technique requires time-consuming sample digestion and has limitations for the analysis of refractory samples, resulting in incomplete digestion. None of these techniques is suitable for the analysis of carbon, which is generally analyzed by using a number of other analytical techniques[6]. Herein, we propose laser induced breakdown spectroscopy (LIBS) as an alternative analytical technique for the analysis of metals and light elements, such as C.

LIBS is very robust for its simplicity and has successfully been used for multi-element analysis, including total carbon. It is advantageous to use LIBS because it enables a rapid in situ sample analysis with little or no sample preparation. The use of an echelle or multi-channel spectrometer gives a broader spectral range allowing for multi-element analysis. Recently, Washburn [9] reported the use of LIBS for geochemical and mineralogical characterization of shale. The spectra from the beginning and the end of laser pyrolysis were used for qualitative analysis of $\mathrm{Mg}, \mathrm{Na}, \mathrm{Li}, \mathrm{K}, \mathrm{Ca}$ and $\mathrm{H}$. No quantification of these elements were reported in his studies.

To the best of our knowledge, no quantitative analysis of the Marcellus shale has been done using LIBS. In this paper, we quantitatively determine major elemental composition of shale rocks by laser induced breakdown spectroscopy. In order to ascertain the quantitative capability of LIBS, our results are compared to those obtained by ICP-OES and a carbon 
analyzer. The elemental concentrations may be mathematically converted to oxide weight percent where necessary for certain geological interpretations.

\section{Experimental set up and sample preparation}

Ten outcrop samples from the Marcellus Shale [10] were used in this study. The powdered samples were pressed into 13-mm diameter pellets using approximately 8-ton pressure, 4 min of dwell time, and 2 min of release time. No binder was added to the pellets. These pelletized shale samples were labeled as S210 - S219.

Measurements were performed using a J200-EC LIBS instrument (Applied Spectra, Fremont, CA) configured with a 266-nm laser and a six-channel optical spectrometer coupled to gated CCD arrays for broadband spectral registration within 190-1040 nm at a resolution of about $0.1 \mathrm{~nm}$. Laser pulse energy was $25 \mathrm{~mJ}$; pulse duration was approximately $4 \mathrm{ns,} \mathrm{while} \mathrm{a}$ flat-top shaped laser beam was collimated onto the sample to produce an ablation spot $150 \mu \mathrm{m}$ in diameter. Laser pulse repetition frequency was $10 \mathrm{~Hz}$. Temporal variation of the Signal-to-noise ratio (SNR) and signal-to-background ratio (SBR) was used to optimize the gate delay. The gate delay with maximum SNR and SBR at $0.2 \mu \mathrm{s}$ was used as the optimal gate delay for sensitive acquisition of both atomic and ionic lines of elements. The gate width was fixed at $1.05 \mathrm{~ms}$. All measurements were performed in air at atmospheric pressure.

The samples were interrogated using a grid of $7 \times 7$ laser ablation spots, covering an overall area of $1.9 \times 1.9 \mathrm{~mm}^{2}$ on the surface of every pellet. Each spot of the grid was ablated with 10 laser pulses and the spectra acquired from these 10 pulses were accumulated. As a result of this interrogation, 49 individual spectra were collected from each sample. The analytical spectral lines are averaged over 49 spectra. These spectra were used to build a multivariate partial least squares model, in order to rectify inter-sample differences while retaining inherent variability of the LIBS signal within every sample.

\section{Results and discussions}

\subsection{Quantitative analysis}

Quantification of elements is performed by producing calibration curves with use of simple linear regression (SLR) and multivariate partial least squares regression (PLS-R). The ten 
samples were divided into two sets, eight for the calibration and two for the prediction sets. It should be noted that some geological analyses may use the independent analyses of samples to generate the SLR and PLS models. To evaluate the figures of merit of LIBS, predictive results from these two approaches are compared to those obtained by ICP-OES and a carbon analyzer. Total carbon was analyzed using a CM5015 Carbon dioxide coulometer (UIC, Inc.) equipped with a high-temperature combustion furnace. Detection limits of these elements are also calculated.

\subsubsection{Spectral lines selection}

From the collected spectra, we were able to identify some major elements in the Marcellus shale notably $\mathrm{Al}, \mathrm{Ca}, \mathrm{Ti}, \mathrm{Si}, \mathrm{Mg}$ and $\mathrm{C}$. These elements corroborated with the previous findings of Heron et al. [11], Gladney et al. [12], and Boström and Bach [13] on rock characterization using neutron induced capture gamma ray spectroscopy, x-ray fluorescence, and inductively coupled plasma optical emission spectrometry. Lines with spectral interference, and resonance lines were mostly avoided and preference was given to non-resonance lines[14]. Quite often, resonance lines are the most affected by self-absorption and self-reversal effects when the concentration or the laser pulse energy is high. These effects are even more pronounced in solid samples than in liquids [15]. For quantitative analysis purposes, the following emission lines from these elements were selected. $\operatorname{Al}(308.21 \mathrm{~nm}, 309.27 \mathrm{~nm}), \mathrm{Ca}(643.90 \mathrm{~nm}, 646.25 \mathrm{~nm}, 649.37 \mathrm{~nm}), \operatorname{Si}(288.15 \mathrm{~nm})$, $\operatorname{Mg}(285.21 \mathrm{~nm}), \operatorname{Ti}(334.94 \mathrm{~nm}, 336.12 \mathrm{~nm}, 337.27 \mathrm{~nm})$ and $\mathrm{C}(247.85 \mathrm{~nm})$ were chosen for simple linear regression (SLR) calibration curves. It should be noted that these are all non-resonance lines except $\mathrm{Al}(308.21 \mathrm{~nm})$ and $\mathrm{Mg}(285.21 \mathrm{~nm})$.

\subsubsection{Simple linear regression}

Figure 1 shows univariate calibration curves of selected analyte lines from which linear regressions are performed for each of the elements using OriginPro 2015 software, manufactured by OriginLab Corporation, USA. 
(a)
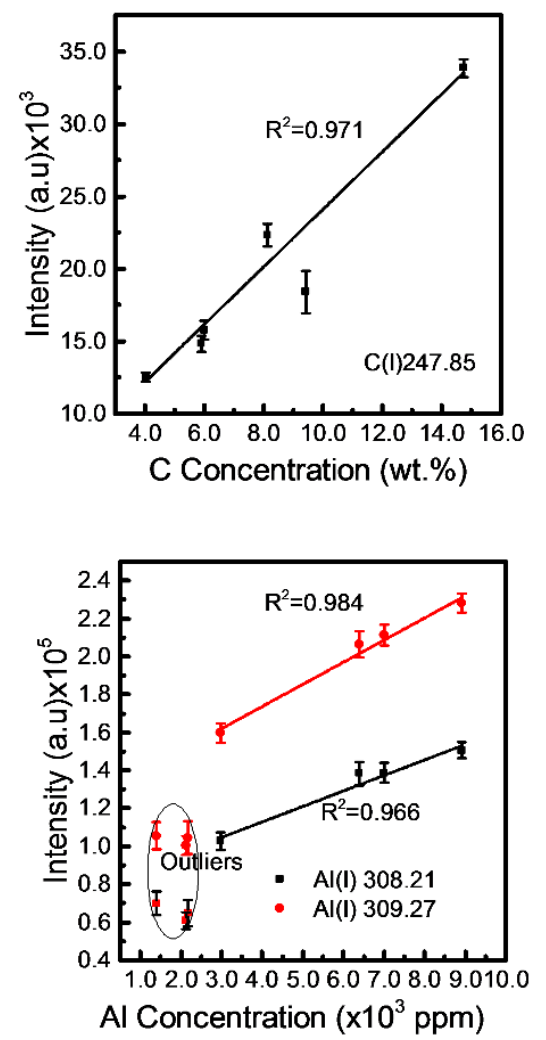

(d) (b)
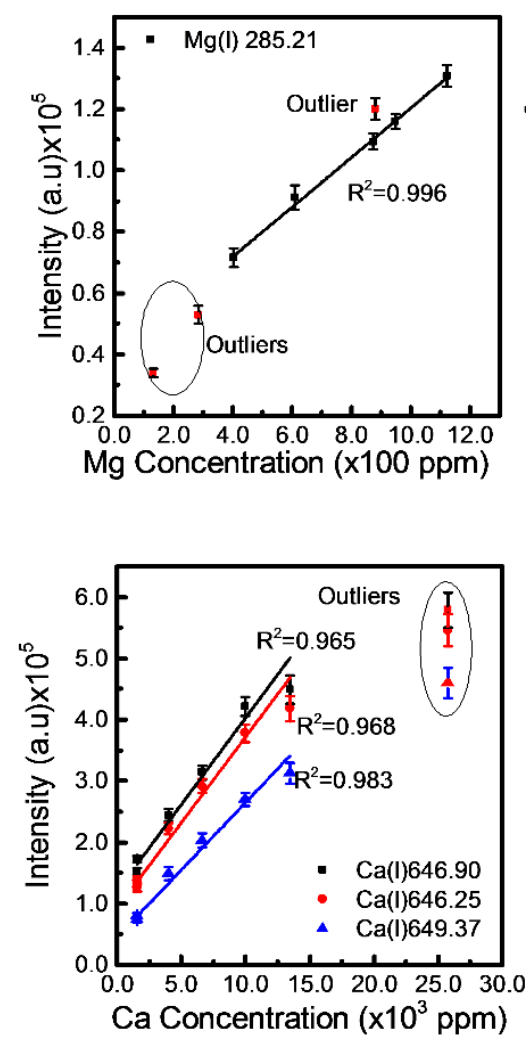

(e) (c)
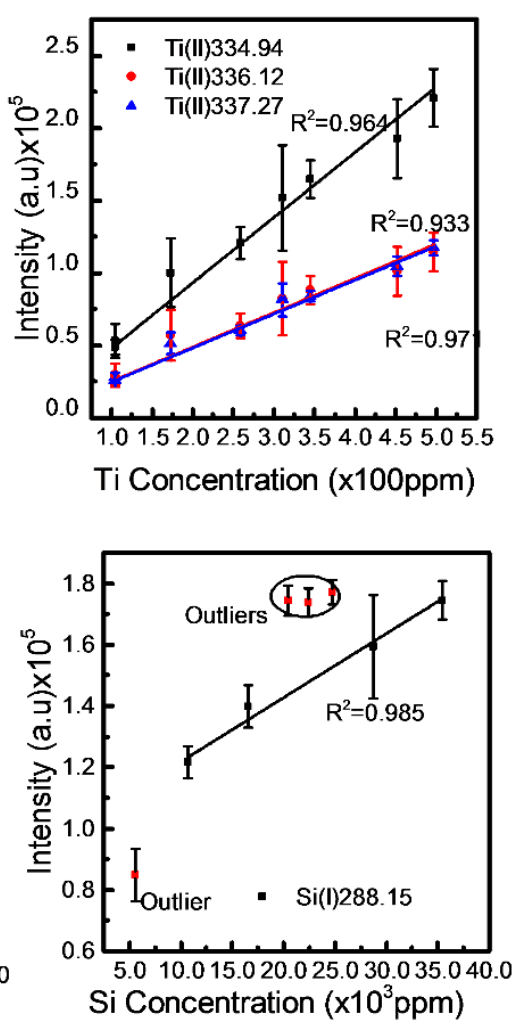

(f)

Figure 1. Simple linear regression calibration curves of selected analyte lines.

The linear regressions in Figure 1 show that $\mathrm{R}^{2}$ is greater than 0.90 , revealing a good correlation of the spectral lines and their concentrations. As seen in the plots in Figure 1, most of the calibration samples lie on the linear fit except for $\mathrm{Al}, \mathrm{Ca}, \mathrm{Si}$ and to a certain degree $\mathrm{C}$ where a few samples cluster away from the regression line and by this, reducing the linear dynamic range for these two elements. The data points lying far from the regression lines are likely outliers. However, the term "outlier" should be used with care. Although these outliers might just be statistical errors in data processing, judging from the relatively small variation of the error bars related to the intensity of these points, they might also be inherent in the very nature of the sample and the LIBS technique, in which case we describe this as "matrix effect." By "matrix effect," we refer to the influence of the physical and chemical properties of the sample on the plasma excitation. Laser-sample interaction and matrix effect have been reported as the main causes of imprecision of LIBS [16]-[18]. Controllable variables including but not limited to choice of analytical line, laser shot-to-shot variance, speed of sample movement, and detector 
settings (time delay and gate width) also affect quantitative analysis of LIBS [17][19]. Specific emphasis on chemical matrix effects has also being discussed by Eppler et al. [20] in studying the effects of chemical speciation and matrix composition on $\mathrm{Pb}$ and $\mathrm{Ba}$ detection in soil and sand samples using LIBS. They found that the chemical compound and sample composition strongly influence emission signals. Since shale rock is composed of different elements with varying concentrations, chemical matrix effects can as well be invoked as source of the outliers observed in the Al, Ca and Si plots (Figure 1,d-f) or spectral interference.

\subsubsection{Use of internal standard to minimize matrix effects}

The nonlinearity of the calibration curve in Figure 1 within certain ranges can also be due to inhomogeneity and multi-elemental content of the shale samples which can induce changes in the plasma excitation. In case the nonlinear calibration curve is due to these changes in excitation, Cremers and Radziemski [16] proposed the use of analyte signal ratioed to another element present in the sample to straighten out the curve. This is known as internal standardization.

In the Al curve (Figure 1d), a linear fit is only observed at high concentrations. The lost sensitivity at high concentrations is often due to self-absorption although saturation of the detector response may also be responsible [16]. At low concentrations, non-resonance line such as $\mathrm{Al}(309.27 \mathrm{~nm})$ are less sensitive to the detector response and if adjacent lines exist near it (which is the case with resonance line $\mathrm{Al}(308.21 \mathrm{~nm})$ ), spectral interference is likely to occur. This might be the case between the aluminum doublets within the 308-309nm spectral range. Consequently, the lines are probably broadened at low concentrations. An attempt to reduce the matrix effect observed in Figure 1d, f was done by normalizing $\mathrm{Al}$ and $\mathrm{Si}$ with $\mathrm{Mg}$ and $\mathrm{Ti}$ respectively. Figure 2 shows the resulting calibration curves. 


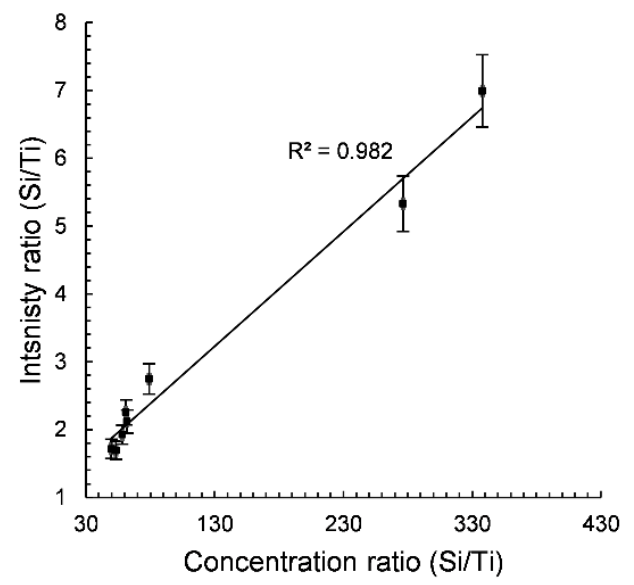

(a)

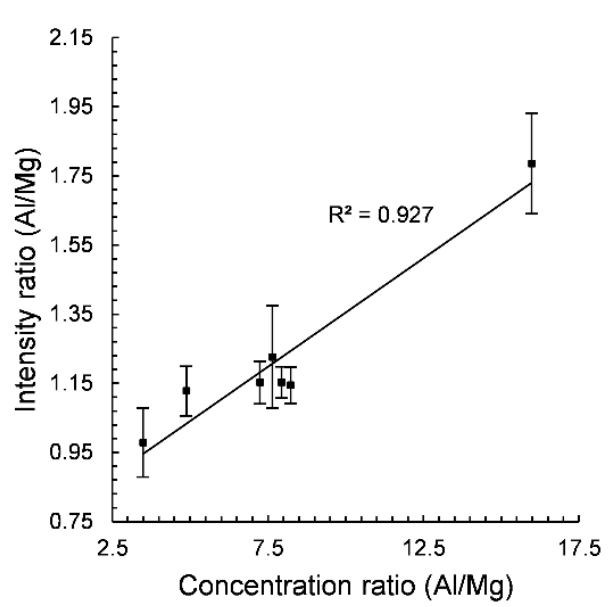

(b)

Figure 2. Calibration curve with internal standards. (a) Si(288.15) / Ti(336.12), (b) Al(308.21)/Mg(285.15)

Calibration curves with internal ratio show $\mathrm{R}^{2}$ greater than that 0.90 over a wider linear dynamic range. In the case of $\mathrm{Ca}$, no element used as an internal standard could yield a better graph. From the results of internal standardization, one can say that the outliers observed in Figure 1d and $\mathrm{f}$ and linked to the influence of other elements notably $\mathrm{Mg}$ and $\mathrm{Ti}$. As for $\mathrm{Ca}$, it is probably related to its high concentration.

It follows that quantitative analysis of a matrix with multiple elements with varying and high concentrations can be affected by spectral interference; and the use of non-resonance lines and intensity ratios can help reduce the matrix effects and improve the quality of simple linear regression calibration curves.

\subsubsection{Partial least squares regression}

An alternative means of minimizing matrix effects is the use of multivariate analysis; this approach is especially useful when we have samples containing emission lines from multiple elements with high likelihood of strong spectral interferences. Multivariate analysis comes in various forms, amongst which is a partial least squares (PLS) analysis. PLS has been widely used to minimize the matrix effect [21][22][23]. PLS provides a model for the relationship between a set of predictor variables $\mathrm{X}$ ( $\mathrm{n}$ objects, $\mathrm{m}$ variables) and a set of response variables $\mathrm{Y}$ ( $n$ objects, $p$ response). In this case, the $m$ variables are the LIBS spectra intensities and the $p$ responses are properties such as the concentration. The $\mathrm{p}$ response has to be independently 
measured for each sample. If the spectral data contain information about the properties of interest, a reliable calibration model can be constructed [24]. The samples with known elemental concentrations are used to create a model relating $\mathrm{Y}$ to $\mathrm{X}$ that is used to predict the concentrations of unknown specimens. PLS has the particularity of reducing the number of variables to a few principal components while taking into account the full spectrum of each sample. Each row of the data matrix $\mathrm{X}$ gives the wavelength-dispersed spectral intensities of one of the calibration samples, and these techniques seek to find a small number of principal component vectors (with the same dimension as the number of columns of $\mathrm{X}$ ) upon which to base the regression. In the case of PLS, the principal component vectors balance the importance of explaining the variance in $\mathrm{X}$ with that of creating a strong correlation with $\mathrm{Y}$ in the regression step described below. This balance results in a robust model with good predictive ability [25][26]. Although PLS often uses the full spectrum for models, some authors have proposed the use of proper selection of spectral ranges for better results when dealing with multi-elemental analysis. $\mathrm{Xu}$ and Schechter used an error indicator function of the net analyte signal to determine the analytical performance of an element in a certain spectral range [21]. Based on this, the most informative spectral ranges to be utilized in multicomponent analysis are selected [27]. Norgaard et al. have proposed interval partial least-squares (iPLS), which consists of developing local PLS models on equidistant subintervals of the full-spectrum region. This method provides an overall picture of the relevant information in different spectral subdivisions, thereby focusing on important spectral regions and removing interferences from other regions [28].

In the present study, the full spectrum was divided into reduced spectral ranges. The reduced spectral range was such that it contains most of the strong lines of a particular element (aluminum for example) for which we want to obtain the calibration curve. This procedure was repeated for others elements $(\mathrm{Ca}, \mathrm{Ti}, \mathrm{Si}, \mathrm{Mg}$ and $\mathrm{C})$. All recorded spectra were used to generate the partial least squares regressions using full cross validation on the average spectra. PLS-R were done with Unscrambler X version 10.3 software, from CAMO Software Inc, USA and the calibration and validation curves are shown in Figure 3. 

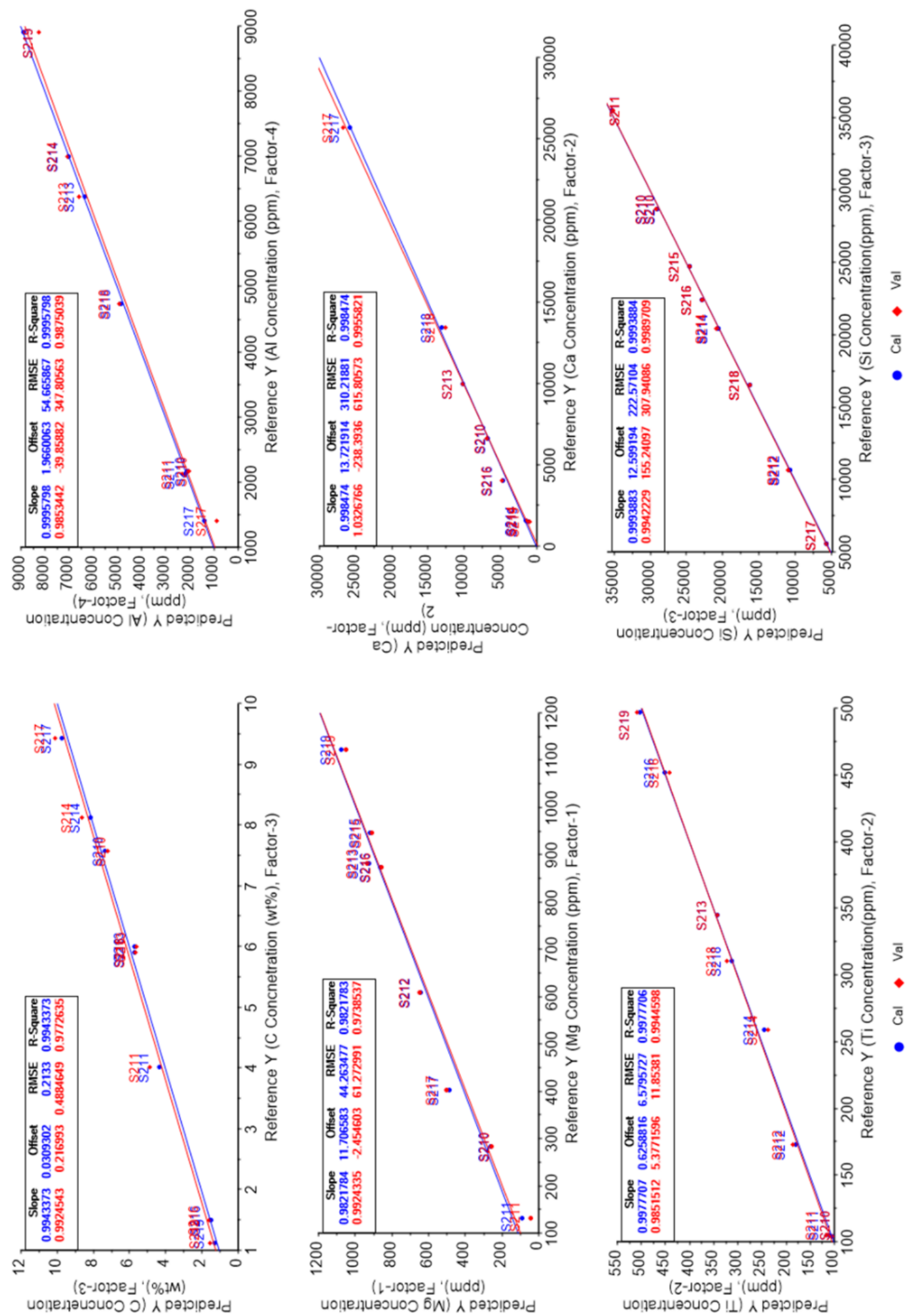

Figure 3. Partial least squares calibration curves. S210-S219 are the samples used for calibration 
In Figure 3, the best fit of the calibration and validation sets deviates slightly from the target line as seen from the values of the slopes which are almost equal to 1 . The $\mathrm{R}^{2}$ values are greater than or equal to 0.98 . These observations show a strong correlation between the predicted and reference values over a wider linear dynamic range than for the univariate method.

\subsubsection{Predictive capability of LIBS}

The predicted concentrations using SLR and PLS-R in our study are compared with those from ICP-OES and a carbon analyzer. The results are reported in Table 1 and a comparison graph is plotted in Figure 4.

Table 1. Prediction results of LIBS and relative errors of determination

Reference values were measured by ICP-OES (Al, Ca, Si, Mg, Ti) and

Carbon analyzer for $C$

\begin{tabular}{cccc|cc}
\hline Predictions & Reference & \multicolumn{2}{c|}{ LIBS } & \multicolumn{2}{c}{ Relative error (\%) } \\
Samples & values & SLR & PLS-R & SLR & PLS-R \\
\hline \multicolumn{5}{c}{$(\mathrm{x} 100 \mathrm{ppm})$} \\
\hline S215_Al & 79.15 & 70.52 & 74.58 & 10.90 & 5.77 \\
S216_Al & 84.69 & 69.23 & 75.26 & 18.25 & 11.13 \\
S218_Al & 47.46 & 34.08 & 48.25 & 28.19 & 1.66 \\
S212_Ca & 171.78 & 167.48 & 156.73 & 2.50 & 8.76 \\
S213_Si & 180.54 & 308.46 & 197.74 & 70.85 & 9.52 \\
S219_Si & 246.88 & 433.66 & 235.63 & 75.65 & 4.55 \\
S214_Mg & 8.51 & 10.09 & 9.35 & 18.56 & 9.87 \\
S218_Mg & 9.33 & 8.27 & 8.25 & 11.36 & 11.57 \\
S215_Ti & 4.06 & 3.29 & 3.55 & 18.96 & 12.56 \\
S217_Ti & 0.96 & 1.38 & 0.52 & 43.75 & 45.83 \\
\hline & & $(w t . \%)$ & & & \\
\hline S210_C & 7.58 & 5.88 & 7.37 & 22.42 & 2.77 \\
S215_C & 3.56 & - & 3.98 & - & 11.79 \\
\hline
\end{tabular}




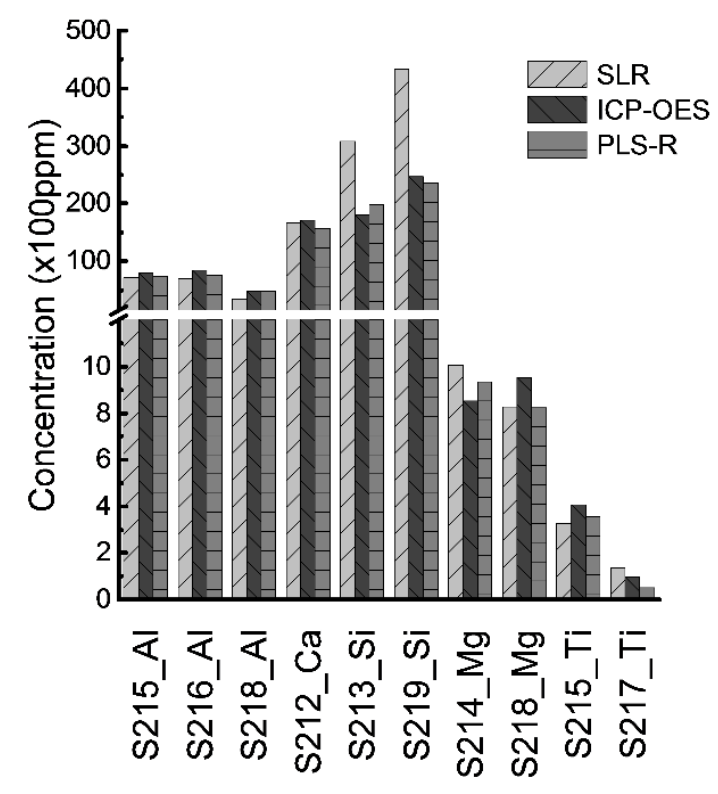

(a)

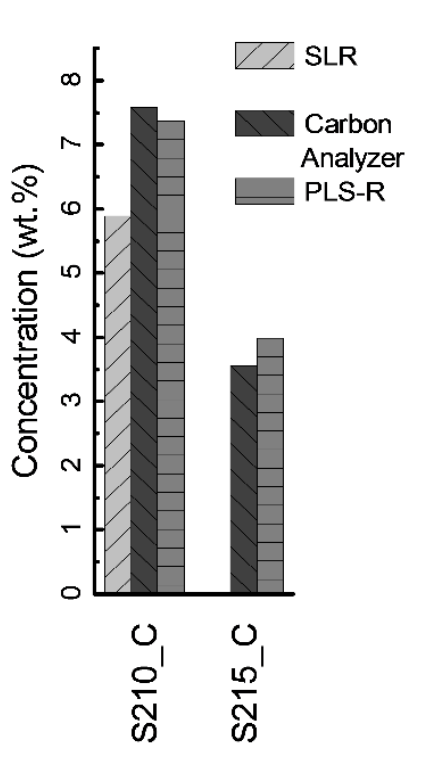

(b)

Figure 4. Comparison of LIBS (SLR and PLS-R) versus ICP-OES and carbon analyzer for Al, Ca, Mg, Si, Ti, and C.

In Table 1, a higher error is noted with SLR prediction of Si. This can be understood from the silicate nature and inhomogeneity of shale rocks. In fact, studies on silicate rocks have shown a high matrix effect of $\mathrm{Si}$ which interferes with $\mathrm{Ti}$ [29]. In order to reduce the sample inhomogeneity, Claisse introduced borate glass [30] to obtain highly reliable and precise results for XRF analysis of all rock types [31]-[33]. The borate fusion method produces homogeneous samples that are easy to handle, can be analyzed multiple times without deterioration, eliminate grainsize and mineralogical effects, and reduce differences in mass absorption from sample to sample [34]. However, LIBS presents a rather simple alternative notably the possibility of avoiding sample preparation, like the case of acid digestion, used to obtain homogeneity of a sample. Although an attempt to reduce matrix effects was made with internal standardization, matrix effect is still present as seen from the SLR calibration, the use of multivariate analysis has been preferred to minimize the matrix effect. In Table 1, the comparison shows that the error for PLS-R (1.66 to $12.56 \%$ ) for most elements is about half that of SLR (2.50 to $75.65 \%$ ). Figure 4 shows that LIBS results are comparable to those of ICP-OES and a carbon analyzer. 


\subsection{Limit of detection}

For each analyte, the spectrum with the lowest concentration was used to calculate its limit of detection (LOD), which is defined as $L O D=3 \frac{c \sigma_{s}}{I}$, where $c$ is the lowest concentration, $\sigma_{s}$ is the standard deviation of the background near the analyzed line, and $I$ the intensity (background subtracted) of the analyzed line. LODs for $\mathrm{Al}, \mathrm{Ca}, \mathrm{Si}, \mathrm{Ti}, \mathrm{Mg}$, and $\mathrm{C}$ are reported in Table 2.

Table 2. Calculated Limits of Detection (LOD)

\begin{tabular}{lc}
\hline \multicolumn{1}{c}{ Elements } & LOD \\
\hline $\mathrm{Ti}$ (II) $336.12 \mathrm{~nm}$ & $15.6 \mathrm{ppm}$ \\
$\mathrm{Mg}$ (I) $285.21 \mathrm{~nm}$ & $4.2 \mathrm{ppm}$ \\
$\mathrm{Al}$ (I) $309.27 \mathrm{~nm}$ & $33.0 \mathrm{ppm}$ \\
$\mathrm{Si}$ (I) $288.15 \mathrm{~nm}$ & $60.9 \mathrm{ppm}$ \\
$\mathrm{Ca}$ (I) $649.37 \mathrm{~nm}$ & $0.03 \mathrm{ppm}$ \\
$\mathrm{C}$ (I) $247.85 \mathrm{~nm}$ & $0.4 \mathrm{wt} . \%$ \\
\hline
\end{tabular}

\section{Conclusions}

Laser induced breakdown spectroscopy (LIBS) was used to determine the elemental composition of Marcellus shale rocks. Major elements ( $\mathrm{Al}, \mathrm{Ca}, \mathrm{Si}, \mathrm{Mg}, \mathrm{Ti}$, and $\mathrm{C}$ ) in shale rocks were qualitatively and quantitatively analyzed. Univariate calibration (SLR) curves of ( $\mathrm{Al}, \mathrm{Ca}$, $\mathrm{Si}, \mathrm{Mg}$, Ti and $\mathrm{C}$ ) were compared to their corresponding multivariate PLS-R calibrations. Quantitative analysis using PLS-R in LIBS helps minimize the matrix effect and increase the linear dynamic range of concentration determination. LIBS offers the possibility of quantifying carbon, which is not possible for analysis by ICP-OES. Predicted results obtained by LIBS are similar to those obtained by ICP-OES for $\mathrm{Al}, \mathrm{Ca}, \mathrm{Si}, \mathrm{Mg}$ and $\mathrm{Ti}$ and by a carbon analyzer for $\mathrm{C}$. From our results and the advantages offered by LIBS (such as its simplified set up, cost and optical emission nature), we have demonstrated that the LIBS method can provide rapid analysis of shale rock samples.

\section{Acknowledgement:}

This research was supported in part by an appointment to the National Energy Technology Laboratory Research Participation Program, sponsored by the U.S. Department of Energy and administered by the Oak Ridge Institute for Science and Education. 


\section{References}

[1] M. Godec, G. Koperna, R. Petrusak, and A. Oudinot, "Enhanced gas recovery and CO2 storage in gas shales: A summary review of its status and potential," Energy Procedia, vol. 63, pp. 5849-5857, 2014.

[2] B. C. Nuttall, C. F. Eble, M. R. Bustin, and J. A. Drahovzal, "Analyses of Devonian Black Shale in Kentucky for Potential Carbon Dioxide Sequestration and Enhanced Natural Gas Production.," Kentucky Geological Survey Report DE-FC26-02NT41442, 2005.

[3] H. T. Schaef, C. L. Davidson, A. T. Owen, Q. R. S. Miller, J. S. Loring, C. J. Thompson, D. H. Bacon, V. A. Glezakou, and B. P. McGrail, "CO2 Utilization and Storage in Shale Gas Reservoirs: Experimental Results and Economic Impacts,” Energy Procedia, vol. 63, pp. 7844-7851, 2014.

[4] A. Busch, S. Alles, Y. Gensterblum, D. Prinz, D. N. Dewhurst, M. D. Raven, H. Stanjek, and B. M. Krooss, "Carbon dioxide storage potential of shales," Int. J. Greenh. Gas Control, vol. 2, no. 3, pp. 297-308, 2008.

[5] K. Piszcz, J. Luczak, and J. Hupka, “MOBILITY OF SHALE DRILL CUTTINGS,” Pysicochemical Probl. Miner. Process., vol. 50, no. 2, pp. 795-810, 2014.

[6] R. A. Nadkarni, "Analytical techniques for characterization of oil shales," Am. Chem. Soc., Div. Pet. Chem., Prepr.;(United states), vol. 28, no. 1, 1983.

[7] P. M. Farkov, L. N. Il'icheva, and a. L. Finkel'shtein, “X-ray Fluorescence Determination of Carbon, Nitrogen, and Oxygen in Fish and Plant Samples,” J. Anal. Chem., vol. 60, no. 5, pp. 426-430, 2005.

[8] I. M. Potgieter, "Analysis of ferromanganese and ferromanganese slag by means of inductively coupled plasma optical emission spectrometry," Appl. Spectrosc., vol. 55, no. 12, pp. 1682-1685, 2001.

[9] K. E. Washburn, "Rapid geochemical and mineralogical characterization of shale by laser-induced breakdown spectroscopy," Org. Geochem., vol. 83-84, pp. 114-117, 2015.

[10] C. W. Noack, J. C. Jain, J. Stegmeier, J. A. Hakala, and A. K. Karamalidis, "Rare earth element geochemistry of outcrop and core samples from the Marcellus Shale," Geochem. Trans, no. 16, pp. 1-11, 2015.

[11] M. M. Herron, M. E. Loan, A. M. Charsky, S. L. Herron, A. E. Pomerantz, and M. Polyakov, "Kerogen content and maturity, mineralogy and clay typing from drifts analysis of cuttings or core," Petrophysics, vol. 55, pp. 435-446, 2014.

[12] E. S. Gladney, D. B. Curtis, and E. T. Jurney, "Multielement analysis of major and minor elements by thermal neutron induced capture gamma-ray spectrometry," J. Radioanal. Chem., vol. 46, no. 2, pp. 299308, 1978.

[13] K. Boström and W. Bach, "TRACE ELEMENT DETERMINATIONS BY X-RAY FLUORESCENCE ANALYSIS : ADVANTAGES,” Proc. Ocean Drill. Program, Sci. Results, vol. 142, pp. 61-68, 1995.

[14] K. J. Grant, G. L. Paul, and J. A. O’Neill, "Quantitative elemental analysis of iron ore by laser-induced breakdown spectroscopy,” Appl. Spectrosc., vol. 45, no. 4, pp. p. 701-705, 1991.

[15] J. P. Singh and S. N. Thakur, "Physics of Plasma in laser-Induced Breakdown Spectroscopy," in LaserInduced Breakdown Spectroscopy, Elsevier, 2007, p. 94.

[16] D. Cremers and L. Radziemski, Handbook of Laser-Induced Breakdown Spectroscopy, 2nd ed. John Wiley \& Sons, 2013.

[17] X. L. Mao, M. a. Shannon, A. J. Fernandez, and R. E. Russo, “Temperature and Emission Spatial Profiles of Laser-Induced Plasmas during Ablation Using Time-Integrated Emission Spectroscopy,” Appl. Spectrosc., vol. 49, no. 7, pp. 1054-1062, Jul. 1995.

[18] J. A. Aguilera, C. Aragón, V. Madurga, and J. Manrique, "Study of matrix effects in laser induced breakdown spectroscopy on metallic samples using plasma characterization by emission spectroscopy," Spectrochim. Acta Part B At. Spectrosc., vol. 64, no. 10, pp. 993-998, Oct. 2009.

[19] I. Bassiotis and A. Diamantopoulou, "Effects of experimental parameters in quantitative analysis of steel 
alloy by laser-induced breakdown spectroscopy,” ... B At. Spectrosc., vol. 56, no. 6, pp. 671-683, Jun. 2001.

[20] A. S. Eppler, D. A. Cremers, D. D. Hickmott, M. J. Ferris, and A. C. Koskelo, "Matrix effects in the detection of $\mathrm{Pb}$ and $\mathrm{Ba}$ in soils using laser-induced breakdown spectroscopy," Appl. Spectrosc., vol. 50, no. 9, pp. 1175-1181, 1996.

[21] J. Amador-Hernández, "Partial least squares regression for problem solving in precious metal analysis by laser induced breakdown spectrometry,” J. Anal. At. Spectrom., vol. 15, no. 6, pp. 587-593, 2000.

[22] S. Clegg, E. Sklute, and M. Dyar, "Multivariate analysis of remote laser-induced breakdown spectroscopy spectra using partial least squares, principal component analysis, and related techniques," Spectrochim. Acta - Part B At. Spectrosc., vol. 64, no. 1, pp. 79-88, Jan. 2009.

[23] S. Laville, M. Sabsabi, and F. R. Doucet, "Multi-elemental analysis of solidified mineral melt samples by Laser-Induced Breakdown Spectroscopy coupled with a linear multivariate calibration," Spectrochim. Acta Part B At. Spectrosc., vol. 62, no. 12, pp. 1557-1566, Dec. 2007.

[24] M. Z. Martin, N. Labbé, T. G. Rials, and S. D. Wullschleger, "Analysis of preservative-treated wood by multivariate analysis of laser-induced breakdown spectroscopy spectra," Spectrochim. Acta Part B At. Spectrosc., vol. 60, no. 7-8, pp. 1179-1185, Aug. 2005.

[25] H. Martens, Multivariate calibration. John Wiley \& Sons, 1992.

[26] C. B. Stipe, B. D. Hensley, J. L. Boersema, and S. G. Buckley, "Laser-induced breakdown spectroscopy of steel: a comparison of univariate and multivariate calibration methods.," Appl. Spectrosc., vol. 64, no. 2, pp. 154-60, Mar. 2010.

[27] L. Xu and I. Schechter, "Wavelength Selection for Simultaneous Spectroscopic Analysis. Experimental and Theoretical Study," Anal. Chem., vol. 68, no. 14, pp. 2392-2400, 1996.

[28] L. Norgaard, J. Wagner, J. P. Nielsen, L. Munc, and S. B. Engelsen, "nterval Partial Least-Squares Regression ( iPLS ): A Comparative Chemometric Study with an Example from Near Infrared Spectroscopy,” Appl. Spectrosc., vol. 54, no. 3, pp. 413-419, 2000.

[29] P. J. Potts, A handbook of Silicate Rock Analysis. Springer Netherlands, 2013.

[30] F. Claisse, "Quebec Department of Mines.," PR 327, 1956.

[31] K. Norrish and B. W. Chappell, "X-ray fluorescence spectrometry," in Physical Methods in Determinative Mineralogy, Academic Press, London, 1967, pp. 161-214.

[32] K. Norrish and B. W. Chappell, "X-ray fluorescence spectrometry," in Physical Methods in Determinative Mineralogy, 2nd ed., Academic Press, London, 1977, pp. 201-272.

[33] K. Norrish and J. . Hotton, "An accurate X-ray spectrographic method for the analysis of a wide range of geological samples," Geochim. Cosmochim. Acta, vol. 33, no. 4, pp. 431-453, 1969.

[34] T. E. La Tour, “Analysis of Rocks Using X-Ray Fluorescence Spectrometry,” Rigaku J., vol. 6, no. 1, pp. 39, 1987. 